\title{
Investigation and Countermeasure Analysis of Nurses' Pressure in the Operating Room of the Third Grade a Hospital in Our City
}

\author{
He Liang, Xuelian Wei, Bo Ma
}

The Third Affiliated Hospital of Qiqihar Medical University, Qiqihar, Heilongjiang, 161000

Keywords: Tertiary hospital; operating room nurse; stress; investigation

\begin{abstract}
To understand the pressure of nurses in the operating room of Grade 3 A hospital in Qiqihar City, analyze the factors, relieve the pressure of nurses and improve the quality of nursing, a total of 300 nurses in operation rooms in five comprehensive tertiary hospitals in Qiqihar City were selected as the survey subjects. This questionnaire was randomly distributed. A total of 300 questionnaires were distributed and 288 were recalled. The recovery rate was approximately $96.0 \%$. The operating room is an important medical resource of the hospital. Through the analysis of the pressure source of the nurses in the operating room, it is proposed that preventive measures should be taken. It is concluded that the managers take active and effective management methods to ease the work pressure of the operating room nurses and improve the quality of care.
\end{abstract}

\section{Introduction}

With the transformation of the medical model, the continuous expansion of the scope of nursing work, the advancement of medical technology, the wide application of new scientific instruments and equipment in the operating room, and the development of surgical models to more advanced, expanding the scope and difficulty of operating room nursing work. Bringing new challenges and greater pressure to the operating room nurses. Stress is a series of physiological and psychological stress response states caused by individual's cognitive evaluation of internal and external environmental stimuli (1). This topic researches the working pressure, coping styles and correlations of the operating room nurses so that nurses in the operating room can correctly understand the pressure and stress sources, build a good coping style in response to various pressures, and promote the physical and mental health of the operating room nurses. Development to ensure that the quality of care in the operating room is further improved.

\section{Research Objects and Methods}

A total of 300 nurses in operation rooms in five comprehensive tertiary-grade A hospitals in Qiqihar City were selected, including 253 female nurses and 47 male nurses; aged 23 to 50 years and 1 to 30 years of age. There are 60 undergraduates, 123 college graduates, and 117 technical secondary schools. Among them, 106 are assistant nurses, 117 nurses, 27 nurses, 42 nurses in charge, and 8 deputy chief nurses.

A questionnaire survey method was used to select part of the contents of the Nurse Work Stress Inventory Scale [2] and the Mental Symptoms Self-Assessment Scale (SCL-90 China Revision Version) [3]. Among them, the content of the questionnaire includes 14 kinds of stress sources investigation and mental health including 9 factors such as somatization, interpersonal sensitivity, obsessive-compulsive symptoms, depression, anxiety, mental disorders, hostility, paranoia, and terror. The content of self-scoring 1 to 5 rating method, the final value of the total score is lower than the psychological level inversely, that is, the total score is higher than the psychological level is low. The questionnaire was distributed randomly. A total of 300 questionnaires were distributed and 288 were recovered. The recovery rate was about $96.0 \%$.

SPSS20.0 statistical software was used for statistical analysis. The measurement data was expressed as $\mathrm{x} \pm \mathrm{s}$. The t-test was performed. The count data were analyzed using the $\chi 2$ test. The test level $\mathrm{p} \square 0.05$ was considered statistically significant. 


\section{Results}

The occupational stressors of operating room nurses (see Table 1).

Table 1 Influence Factors of Occupational Stress of Operating Room Nurses

\begin{tabular}{|c|c|c|}
\hline factor & Number of cases & percentage (\%) \\
\hline Work burden & 234 & 81.2 \\
\hline Management factors & 230 & 79.9 \\
\hline Role pressure & 206 & 71.5 \\
\hline Human relations & 180 & 62.5 \\
\hline $\begin{array}{c}\text { Bad working } \\
\text { environment }\end{array}$ & 174 & 60.4 \\
\hline Nurse's own factors & 169 & 58.7 \\
\hline
\end{tabular}

SCL-90 scores for operating room nurses (see Table 2).

Table 2 Scores of SCL-90 Factors in Operating Room Nurses

\begin{tabular}{|c|c|c|c|c|c|c|}
\hline factor & \multicolumn{7}{|c|}{ factor Score(Case) } \\
\hline & $\mathbf{2}$ & $\mathbf{2 . 5}$ & $\mathbf{2 3}$ & $\mathbf{2 3 . 5}$ & $\mathbf{2 4}$ & $\mathbf{\mathbf { 4 . 5 }}$ \\
\hline Somatization & 43 & 8 & 38 & 178 & 18 & 3 \\
\hline Anxiety & 38 & 33 & 113 & 78 & 26 & 0 \\
\hline depressed 7 & 3 & 123 & 48 & 33 & 8 & 3 \\
\hline force & 74 & 87 & 86 & 41 & 0 & 0 \\
\hline
\end{tabular}

\section{Discussion}

The work rhythm is relatively fast, requiring a high concentration of energy; labor intensity is high, the physical fitness of the nurse is required to be higher; the life is irregular, and the precision is high.

The operating nature of the operating room forced the operating room nurse to be in a state of intense mental tension and severe overdraft for a long period of time, which led to the operating room nurse being in an excessively intense and high pressure state at all times.

The operating room is a relatively closed working environment. This kind of work environment also increases the work pressure of the operating room nurse. Operating room nurses are often exposed to a noisy working environment, monitoring noise, incisions produced by electric knife cutting tissue, corrosive and irritating chemicals, and radiation radiation hazards, all of which have an invisible psychological impact on operating room nurses. Work pressure.

Nurses who work in the operating room for a long time often come into contact with various chemical agents, especially those that have strong contaminating and teratogenic effects. The operating room has a large number of patients undergoing surgery every day. The patients suffer from poor physical function, complicated diseases, various causes, and need to be in close contact with the patients. They produce various secretions such as body fluids, saliva, sputum, and blood from patients entering and leaving the operating room. All substances are highly contagious and seriously affect the health and safety of the nurses in the operating room.

In the operating room, anesthesia and surgery coexist in one room. The anesthesia produces anesthetic induction noise caused by instruments and equipment such as exhaust gas, anesthesia monitor, etc. These factors affect the physical and mental health of the nurses in the operating room for a long time. 
The operating room nurses are in different roles at work and in the family. Due to the high risk of work, high pressure and other factors, the negative impact of the work affects the emotions in the family. Not only does not have too much time to accompany the family, it can easily affect the harmony of the family, and then bring the contradictions and emotions in the family to work, affect the work, causing unnecessary clinical errors.

Reasonably adjust workload and work intensity. Operating room nurses have a high-intensity and overloaded working condition, which is often caused by a shortage of staff in the operating room. High-intensity and over-loading work has been already too exhausted and high-stressed can not fully rest and ease, making the nurse physically and mentally exhausted, which seriously affects the operating room nurses work quality and work efficiency. For the phenomenon of high-intensity and overloaded working conditions, the head nurse should make reasonable arrangements for rest time and overall arrangement of human resources, and timely arrange group collaborative games and activities, which can not only stabilize the team's cohesion but also make the operating room nurses physically and mentally aware. Fully relaxed to be able to work better.

Strengthen humane management and relieve pressure on nurses. The head nurse, as a manager, should perform targeted personnel management in accordance with the job title, age, and academic qualifications of the nurses in the clinical nursing work. The establishment of an incentive mechanism to increase the sense of identity of the work, and appropriately increase the establishment of nurses, flexible and flexible schedule. Appropriately increase the chances of promotion of operating room nurses, improve nurses' wages and benefits, improve the social status of nurses, and make nurses psychologically satisfied.

Actively optimize the working environment and management system. Operating room environment is relatively closed, due to surgical needs, in order to prevent dust and flying insects into most designs without windows, and according to different surgical needs, the operating room area is limited to $20 \sim 50 \mathrm{~m} 2$, reasonable layout in a limited space. Nurses must not only work with the surgeon, but also observe the condition of the patient, manage the use of medicines and devices, have a high concentration of mental state, and the operating room tends to be confined. The long-term confined space will cause people to feel anxious, if not timely. Control and regulation is a serious psychological factor. The operating room is a relatively special and closed working environment. This is an unchangeable working environment. For such a working environment, we must increase the frequency of ventilation in the operating room, avoid harmful gas residues, and reduce harmful gases to the operating room staff and patients. s damage.

Regular inspection and maintenance of operating room monitors, anesthesia equipment and other equipment to ensure that all types of equipment in the best working condition, reduce the operating noise of various instruments to ensure the operating room environment in the best condition. The nurses at all levels of the operating room have clear division of labor and responsibility. The head of the operating room gives full play to the leadership role, supervises and inspects the daily work of the nurses in the operating room, organizes business learning and technical assessments, and improves the performance of the operating room nurses and doctors and patients. The relationship continues to improve the nursing model and formulate a responsibility system for nursing care.

Improve the quality of nurses and their awareness of occupational hazards. With the development of science and technology and the renewal of knowledge, the use of new technologies in clinical surgery, and the use of new equipment in use have become more intense. Nurses need to continuously learn, update their knowledge framework and master clinical skills in order to adapt to the new environment brought by scientific and technological advancement and to meet medical needs. The new requirements, the hospital should establish a sound operating room rules and physique, develop a set of reasonable operating room nursing quantitative assessment rules, in the work of continuous self-control, self-correction, and only in this way can comprehensively improve the overall quality of nurses [4] . Organize regular learning on the prevention and control of occupational hazards to prevent unnecessary harm from happening. The hospital should establish a health record for each nurse [5] and arrange a targeted physical examination once a year.

In order to improve the work efficiency of operating room nurses, to protect the physical and 
mental health of nurses, and to address the threat of acute injury and blood-borne transmission diseases in the operating room, hospitals should strengthen the training of occupational safety education and protection knowledge and skills for junior nurses. At the same time, reasonable arrangements for operating personnel, the establishment of nurses health records, sharp injury reporting registration system, sharp injury prevention and treatment system and sharp injury treatment process, so that nurses in the work of the rules to follow, there are laws to follow.

In view of the large amount of surgery, long time, and many health hazards, a more reasonable duty shift system should be established to effectively ensure the rest time of nurses and related medical personnel. It should maximize the need for perspective or radiography procedures. Reduce the distance between radiation sources and nurses, increase the investment in lead barriers and lead protective clothing, and minimize the radiation exposure time of medical personnel.

The operating room is an important medical resource for the hospital. Through the analysis of the pressure source of the operating room nurses, the prevention measures are intervened. It is concluded that the managers take active and effective management methods to ease the work pressure of the operating room nurses, so that the nurses are fully dedicated to work. , Shorten the operation time, reduce wastage of surgical consumables, familiarize with new surgical equipment, new materials, increase the technical content of surgery, reduce costs, and accelerate the turnover rate of surgery, thereby improving the efficiency and effectiveness of the operating room, shortening the average hospital stay, and reducing patients economic burden.

\section{Acknowledgements}

Fund Project: Soft Science Research Project in Qiqihar City: Analysis of the nurses' stressors in the operating room of the top three hospitals in Qiqihar City and study of preventive measures (Project No. RKX-201526)

\section{References}

[1] Li Xiaomei, Feng Xianqiong. Introduction to Nursing (Fourth Edition). Beijing: People's Medical Publishing House, 2017.

[2] Li Xiaomei. Introduction to nursing [M]. Changsha: Hunan Science and Technology Press, 2001.

[3] Li Xiaomin. Hidden dangers and countermeasures of nursing care in operating room[J]. China Practical Medicine, 2009, 4(33): 201-202.

[4] Feng Defang. Lu Jingmei. Occupational safety hidden troubles in the operating room and preventive measures [J]. Chinese community physicians, 2010,12 (11).

[5] Tang Yan, Liu Xiaoyun, Zhang Xiaojiao. Investigation and Analysis of Nurses' Work Stress Sources and Related Factors [J]. Nursing Research, 2008, 22(1): 202-203. 